\title{
Recovery Interval during Physical Training Depends on both Neural and Energetic Constraints
}

\section{José Augusto Rodrigues dos Santos* and Alcino da Silva Carvalho}

Faculty of Sports, University of Porto, Portugal

\section{Introduction}

Physical training and sport conditioning depends on the correct conjugation of stimulus-load, resting and nutrition. Given that load and nutrition are adequate for training goals, rest handling is crucial for training control and posterior adaptation. Adequate resting between exercises, sets or workouts gives the direction for muscle/ body adaptation, allowing not only to achieve training goals but also to avoid situations of extreme fatigue whose deficient copping can induce non-physiological overreaching or pathological overtraining [1]. Thus, analysing optimal resting intervals is of surmount importance in the training process of different activities as high level sport training and rehabilitation.

\section{The logic of interval in sports training}

We have previously observed that the level of fatigue induced by three consecutive training days was well tolerated by recreationally trained subjects and induced similar results on maximum strength and body composition than normal periodization that respects a $48 \mathrm{~h}$ interval between resistance training sessions [2]. This information is useful both for recreational and elite athletes, and even for people with neurologic constraints. The main problem to solve after three consecutive training days is the control of the stress imposed by the exercises load (volume and intensity) that should be very well monitored. Therefore, subjective fatigue assessed by the Recovery-Stress Questionnaire for Athletes [3] or physiological fatigue assessed by different biomarkers (e.g. creatine kinase, cortisol, ammonia and creatine) [4] should be periodically controlled.

The capability for training in consecutive days maintaining high exercise loads open new possibilities for time management on innovative training protocols. Well managing time is fundamental for the recovery process in people with neural injuries, and training continuity on a daily basis can be the best solution for some neural and cognitive impairments [5], evidencing the importance to concentrate overload periods counterbalanced with adequate rest.

It is well known that sustained physical exertion leads to a reduced capacity to produce voluntary force through different mechanisms [6]: (i) suboptimal cortical drive; (ii) reduced synaptic efficacy; (iii) release of neuromodulators (e.g. serotonin); (iv) reduced intrinsic moto-neuron responsiveness; (v) presynaptic inhibition; (vi) exaggerated firing of III/ IV muscle afferents; (vii) excitation-contraction coupling failure; and (viii) reduced efficacy on cross-bridge effects. These mechanisms impair muscle function or reduce central nervous system capability to activate skeletal muscles (peripheral and central fatigue, respectively), with both being usually recovered within $24 \mathrm{~h}$ if loads are individually adequate and proper nutrition is provided.

However, it is very difficult to ascertain when acute fatigue and marginal energetic deficits are translated into chronic physical and neurological impairment. Training process should be seen as a whole and the two main systems (brain and immune system) compete for energy and have prolonged recovery times [7]. The brain has a central position amongst all the organs concerning energy metabolism as it controls energy supply and receives information from peripheral sensors and their afferent neuronal pathways. As the brain is a high energy "consumer", but its capacity to store energy is very limited, its energetic turnover is fundamental for survival [8] and its metabolic recovery capital for exercise adaptation. The immune system has a high rate of cell proliferation and requires a rapid turnover of proteins. It is extremely vulnerable to energy deficits and recovery time is fundamental not only for energetic purposes but also for controlling exercise-induced inflammation [9].

As energy imbalance or inadequate recovery time can negatively affect the planned adaptations, physical loads during training should be well controlled through different biochemical (e.g. cortisol, creatine kinase, lactate and homocysteine), physical (e.g. strength and power) and neurocognitive (e.g. Brain Derived Neurotrophic Factor Gene (BDNF), Vascular Endothelial Growth Factor (VEGF) and FGF2 fibroblast growth factor 2 (FGF-2) ) markers. Here, once more, the solution is individual and supported by common sense. For high level sport and neural rehabilitation, even having the risk of overtraining or pathological overreaching, intensification of training loads (increasing exercise's intensity and volume or decreasing recovery time) can be the way for high level performances. For recreational purposes (i.e. for health and well-being), training loads should be directed not for maximal performance but towards a stabilized level of physical conditioning that permits a good functional reserve for everyday life.

Our study verified that fatigue level induced by three consecutive training days is well supported if a large resting period is respected. Training efficacy is always a question of load and recovery. In relation to a single load, for instance, a brief high-intensity exercise (e.g. a burst of $8 \mathrm{~s}$ sprint running), recovery time to offset central (neural) fatigue lasts no more than 2-3 min, while peripheral fatigue takes usually 3-5 min to recovery [6]. However, a training program includes different exercises repeated several times that induce increasingly levels of fatigue and impairment on muscle function requiring a long resting period for full recovery. From our work it can be stated that even if full recovery is not reached on a daily basis, fatigue summation was well tolerated by the athletes and higher performance levels were attained when sufficient rest is respected between trained phases. It is important to highlight that this is true for recreationally athletes and that it is necessary to ascertain if our protocol is feasible and adequately integrated in more exigent training protocols.

*Corresponding author: José Augusto Rodrigues dos Santos, Faculty of Sport, University of Porto, Portugal; E-mail: jaugusto@fade.up.pt

Received October 31, 2018; Accepted January 07, 2019; Published January 14 2019

Citation: dos Santos JAR, da Silva Carvalho A (2019) Recovery Interval during Physical Training Depends on both Neural and Energetic Constraints. Int J Neurorehabilitation 6: 337. doi: 10.4172/2376-0281.1000337

Copyright: (c) 2019 dos Santos JAR, et al. This is an open-access article distributed under the terms of the Creative Commons Attribution License, which permits unrestricted use, distribution, and reproduction in any medium, provided the original author and source are credited. 
Citation: dos Santos JAR, da Silva Carvalho A (2019) Recovery Interval during Physical Training Depends on both Neural and Energetic Constraints. Int J Neurorehabilitation 6: 337. doi: 10.4172/2376-0281.1000337

\section{References}

1. Meeusen R, Duclos M, Foster C, Fry A, Gleeson M, et al. (2013) Prevention, diagnosis and treatment of the overtraining syndrome: Joint consensus statement of the European College of Sport Science and the American College of Sports Medicine. Med Sci Sports Exerc 45: 186-205.

2. da Silva Carvalho AD, Rodrigues Santos JA (2018) Nonconsecutive versus consecutive-day resistance training in recreationally trained subjects. J Sports Med Phys Fitness 58: 233-240.

3. Kellman M (2010) Preventing overtraining in athletes in high-intensity sports and stress-recovery monitoring. Scand J Med Sci Sports 20: 95-102.

4. Palacios G, Pedrero-Chamizo R, Palacios N, Maroto-Sánchez B, Aznar S, et al. (2015) Biomarkers of physical activity and exercise. Nutr Hosp 31: 237-244.

5. Tsai CL, Ukropec J, Ukropcová B, Pai MC (2017) An acute bout of aerobic or strength exercise specifically modifies circulating exerkine levels and neurocognitive functions in elderly individuals with mild cognitive impaitment. Neuroimage Clin 17: 272-284.

6. Carroll TJ, Taylor JL, Gandevia SC (2017) Recovery of central and peripheral neuromuscular fatigue after exercise. J Appl Physiol 122: 1068-1076.

7. Yamagata AS, Mansur RB, Rizzo LB, Rosenstock T, Mclntyre RS, et al. (2017) Selfish brain and selfish immune system interplay: A theoretical framework for metabolic comorbidities of mood disorders. Neurosci Biobehav Rev 72: 43-49.

8. Ye Q, Wu Y, Gao Y, Li Z, Li W, et al. (2016) The "selfish brain" is regulated by aquaporins and autophagy under nutrient deprivation. Mol Med Rep 13 3842-3848.

9. Griffiths HR, Gao D, Pararasa C (2017) Redox regulation in metabolic programming and inflammation. Redox Biol 12: 50-57. 